\title{
Estimation of Power Imbalance Size with Consideration of Impact of Emulated Inertia
}

\author{
Qiteng Hong and Campbell Booth \\ Institute for Energy and Environment, \\ University of Strathclyde, \\ Glasgow, UK
}

\author{
Bin Wang and Xinzhou Dong \\ Department of Electrical Engineering, \\ Tsinghua University, \\ Beijing, China
}

\author{
Liang Ji \\ School of Electrical Power Engineering, \\ Shanghai University of Electric Power, \\ Shanghai, China
}

\begin{abstract}
Accurate estimation of power imbalance (e.g. loss of generation size) during frequency disturbance events is of vital importance for effective power system monitoring and control. Conventional methods for power imbalance estimation are mainly based on the swing equation, where the size of power imbalance is calculated using the measured Rate of Change of Frequency (RoCoF) and the inertia level of the system. With the increasing penetration of renewable generation, the system inertia has decreased significantly. Inertia emulation is considered as a promising solution for enhancing frequency stability in future power systems. However, the emulated inertia, acting in a similar manner of true inertia, could have an impact on the measured RoCoF, which will subsequently affect the accuracy of the power imbalance estimation using conventional methods. Therefore, this paper presents a comprehensive evaluation of the impact of such emulated inertial response on the RoCoF behaviour during frequency disturbances, thus the impact on the accuracy of power imbalance estimation. A new power imbalance estimation method that takes into account of the impact of emulated inertia is proposed. Case studies using both an analytical model and a microgrid model simulated in a real-time simulator are presented, which demonstrate that the proposed power imbalance estimation method could achieve significantly higher estimation accuracy compared to conventional methods, thus offering an effective solution for estimating frequency disturbance sizes in systems with emulated inertia.
\end{abstract}

\section{INTRODUCTION}

Power systems will typically experience frequency disturbances when there are power imbalances (i.e. imbalance of generation and demand), which could be caused by loss of generation/infeed or loads. Accurate estimation of the size of power imbalance (referred to as event estimation for simplicity) in power systems is of vital importance for effective monitoring and subsequent control actions to restore the frequency [1], [2]. Conventional methods for event size estimation are mainly based on the swing equation, where the inertia level and the measured RoCoF are used to calculate the power imbalance [3], [4].

With the increasing penetration of renewable generation, the overall system inertia has decreased significantly [5]. Inertia emulation has been proposed as a promising solution for enhancing frequency stability in future power systems [6]. However, the emulated inertia, acting in a similar manner of the true inertia, could have an impact on the measured RoCoF, which will subsequently affect the accuracy of the event estimation using conventional methods.
Therefore, this paper presents a comprehensive evaluation of the impact of the emulated inertial response on the measured RoCoF during frequency disturbances, thus the impact on the estimation accuracy. A new event estimation method that takes into account of the impact of emulated inertia is presented. The proposed event estimation method uses the estimated inertial response power to compensate the event size calculated using conventional methods, which is capable of achieving significantly higher estimation accuracy in power systems with emulated inertia.

The paper is organized as follows: Section II introduces the conventional methods that have been widely used for event size estimation and their limitations; Section III presents the proposed event estimation method; Section IV presents a number of case studies that demonstrate the effectiveness of the proposed method; and Section V concludes the paper and highlights the future work.

\section{CONVEnTIONAL EVENT SIZE ESTIMATION METHODS AND THEIR LIMITATIONS}

\section{A. Fundamentals of conventional methods}

The conventional methods for event size estimation as reported in [2]-[4] are typically based on the swing equation as presented in (1) [7]:

$$
\frac{2 \sum H_{S M}^{i} S_{S M}^{i}}{f_{n}} \times f_{p u} \times R o C o F(t)=\Delta P(t)
$$

where $H_{S M}^{i}$ and $S_{S M}^{i}$ are the inertia constant and the rating of the $i^{t h}$ synchronous machine in the system respectively; $\mathrm{RoCoF}(t)$ is the Rate of Change of Frequency (RoCoF); $f_{n}$ is the nominal frequency; $f_{p u}$ is the measured pre-event frequency in per unit; and $\Delta P(t)$ is the power imbalance.

To estimate the initial power imbalance when the event occurs $\left(\Delta P_{E}\right)$, the maximum value of the RoCoF $\left(R o C o F_{\max }\right)$ is measured and used for the calculation. Assuming the preevent frequency is of the nominal value, then the size of the power imbalance event $\Delta P_{E}$ can be calculated using (2), which is referred to as the conventional method in the rest of the paper for simplicity.

$$
\Delta P_{E}=\frac{2 \sum H_{S M}^{i} S_{S M}^{i}}{f_{n}} \times R o C o F_{\max }
$$




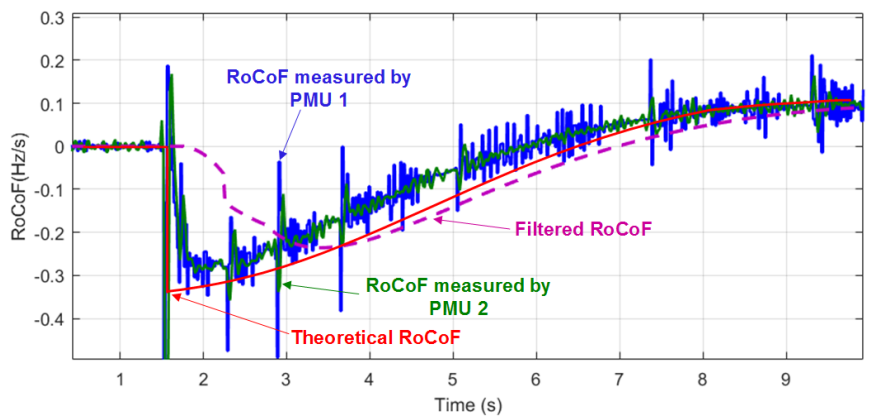

Fig. 1. Comparison of theoretical and measured RoCoF during a frequency disturbance

\section{B. Limitations of the conventional method for systems with emulated inertia}

From (2), it can be seen that the accuracy of the event size estimation is largely dependent on the accuracy of the measured $R o C o F_{\max }$ value. The measurement of RoCoF, particularly during frequency disturbances, can suffer from significant errors [8]. Furthermore, the accuracy of RoCoF measurement is also largely subject to the measurement algorithms, where different measurement approaches could also lead to signifiant variations in the measured RoCoF [8].

Fig. 1 shows the RoCoF from theoretical calculation, measurements of different Phasor Measurement Units (PMUs) and results from low pass filters used for removing the measurement noises. It can be seen that, the RoCoF measurements from PMUs can be very noisy. Therefore, filters are typically required to remove these noises. The time constant of the filter can vary from tens to hundreds of milliseconds. Therefore, the emulated inertia, which typically responds in the scale of milliseconds, will have an impact on the measured and filtered RoCoF, leading to the estimation result not reflecting the true power imbalance size (but a combination of power imbalance size and the emulated inertial response). Therefore, using the conventional method purely relying on the measured RoCoF could lead to significant errors in systems with emulated inertia.

\section{Estimation of Event Size With Consideration OF INERTIA EMULATION}

The proposed new method for power imbalance event size estimation with consideration of the emulated inertia is illustrated in Fig. 2. The method uses the frequency $f$ and RoCoF measurements as input signals. The first step is to determine whether if there is a genuine frequency disturbance through the event detection block, where the measured frequency and $\mathrm{RoCoF}$ are compared with a number of pre-configured thresholds. The frequency thresholds are typically configured as the frequency range under normal operating conditions, which is $49.8 \mathrm{~Hz}$ to $50.2 \mathrm{~Hz}$ in the GB system [5]. For the configuration of the RoCoF thresholds, there is a level of flexibility as for the same measured RoCoF, there could be different amounts of power imbalances (either due to normal load changes or real loss of generation/load events)

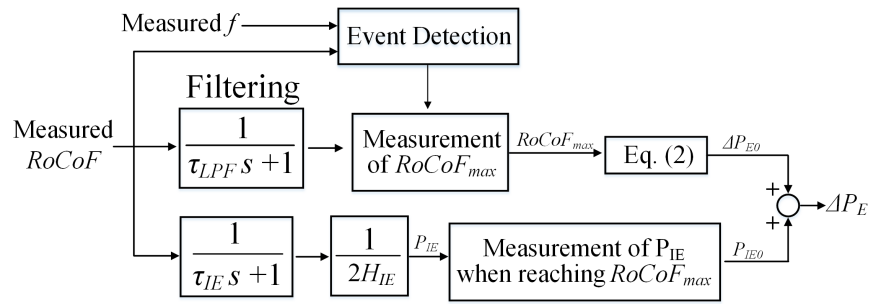

Fig. 2. The proposed method for event size estimation with consideration of emulated inertia

depending on the overall system inertia level. Therefore, the thresholds for RoCoF should be configured based on how sensitive the end users would like the algorithm to detect the disturbances in the system. The event detection block is also included a timer where only the thresholds are violated for a configurable period of time, it will consider there is an event (i.e. a frequency disturbance). This is to avoid mal-detection of events due to transient spikes in the measured data (especially from RoCoF data).

If a frequency disturbance is detected, the event size calculation process will be initiated as shown in Fig. 2. The measured $\mathrm{RoCoF}$ will be firstly filtered to remove undesirable transient spikes and the maximum RoCoF will be measured. Using Eq. 2, $\Delta P_{E 0}$, which is the estimated event size using the conventional method without consideration of inertia emulation can be calculated. In addition to $\Delta P_{E 0}$, the algorithm also estimates the contribution of the power from emulated inertia, which has an impact on the the measured $R o C o F_{\max }$. $P_{I E 0}$ is the estimated inertial response power at the time where RoCoF reaches the maximum value $R o C o F_{\max }$. By adding $P_{I E 0}$ and $\Delta P_{E 0}$, the estimated event size is compensated with the contribution from inertia emulation, thus offering a more accurate estimation result.

\section{CASE STUdY}

In this section, case studies are presented to demonstrate that by using the proposed method for event size estimation with consideration of inertia emulation, the estimation accuracy can be significantly improved compared with the conventional method, which does not take into account of the emulated inertia. The case studies firstly use an analytical system frequency response model and subsequently a microgrid model simulated in a real-time simulator. The reason of using the analytical model is that it allows transparent analysis of the various parameters during the event without being affected by issues such as measurements. The use of the microgrid model is for realistic evaluation of accuracy of the proposed event estimation method.

\section{A. Validation of the proposed event estimation method using an analytical model}

The analytical power system frequency response model is shown in Fig. 3, and it is constructed based on the model reported in [9]. The description of the various parameters is provided in Table I. Using this model, the impact of increasing 


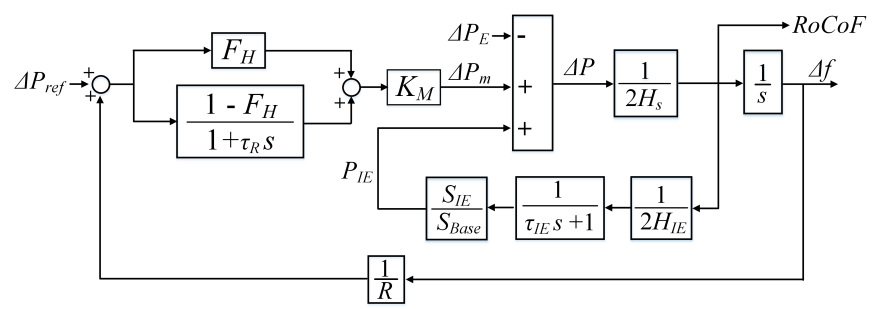

Fig. 3. System frequency analytical model with consideration of inertia emulation [9]

TABLE I

DESCRIPTION OF PARAMETERS IN THE ANALYTICAL MODEL AND THE ASSOCIATED VALUES FOR CASE STUDIES

\begin{tabular}{c|l|c}
\hline Parameter & Description & Value \\
\hline$F_{H}$ & $\begin{array}{l}\text { Fraction of total power generated by the } \\
\text { turbine }\end{array}$ & 0.1 \\
\hline$\tau_{R}$ & Reheat time constant & 8 \\
\hline$K_{M}$ & Mechanical power gain factor & 0.95 \\
\hline$H_{s}$ & $\begin{array}{l}\text { System equivalent inertia constant in sec- } \\
\text { onds }\end{array}$ & $2 \mathrm{~s}$ \\
\hline$\tau_{I E}$ & Inertia emulation time constant & 0.1 \\
\hline$H_{I E}$ & Emulated inertia constant in seconds & $6 \mathrm{~s}$ \\
\hline $\mathrm{R}$ & Regulation constant for droop control & 0.05 \\
\hline$P_{I E}$ & Active power from inertia emulation in pu & - \\
\hline$\Delta P_{E}$ & Actual power imbalance size in pu & $0.3 \mathrm{pu}$ \\
\hline$S_{B a s e}$ & Base power in MVA & $3 \mathrm{MVA}$ \\
\hline$S_{I E}$ & $\begin{array}{l}\text { Capacity of resources providing inertia em- } \\
\text { ulation in MVA }\end{array}$ & $1 \mathrm{MVA}$ \\
\hline
\end{tabular}

inertia emulation on the estimation of accuracy of the event size can be evaluated.

Fig. 4 presents the simulation results of a study using the analytical model with the parameters' values presented in Table I, where the first plot shows the simulated frequency; the second plot presents the simulated non-filtered and filtered RoCoF; the third plot presents the power output from inertia emulation; the fourth and fifth plots show the estimation of power imbalance size using the conventional method and the proposed new method respectively; and the last plot compares the event size estimation error using the conventional method and the proposed new method.

In Fig. 4.(a), there is no inertia emulation enabled, so $P_{I E}$ is zero through out the simulation. A power imbalance event with a size of $0.3 \mathrm{pu}$ is triggered at $1 \mathrm{~s}$. Since there is no inertia emulation, it can be seen that the estimation errors for using both methods are similar. These errors are mainly caused by the filtering of RoCoF to remove measurement noises. In this case, because an analytical model is used, there is no obvious noise in the measured RoCoF.

Fig. 4.(b) shows the simulation results when inertia emulation is enabled with $H_{I E}=6$ and $S_{I E}=1 \mathrm{MVA}$. In this case, it can be seen that there is a clear improvement in the estimation accuracy using the proposed method - the estimation error using the conventional method is approximately $0.1 \mathrm{pu}$ while the error using the proposed method is approximately $0.01 \mathrm{pu}$.

Fig. 5 shows the comparison of estimation errors between the conventional method and the proposed method with the increase of $H_{I E}$ and $S_{I E}$. As it can be seen that, with the increasing values of the emulated inertia constant $H_{I E}$ and the capacity of the source $S_{I E}$ providing inertia emulation, the estimation error using the conventional method can increase significantly. The increase of $H_{I E}$ and $S_{I E}$ effectively contributes to the increase of the impact of emulated inertia on the measured RoCoF, thus affecting the event size estimation accuracy. In contract, using the proposed event estimation method, the error is generally lower than the conventional method and remains largely unchanged with the increase of $H_{I E}$ and $S_{I E}$. These results show that the proposed method can achieve higher event estimation accuracy, while being largely immune to the level of inertia emulation deployed in the system.

\section{B. Validation of the proposed event estimation method using a microgrid model}

The section presents the validation of the proposed event estimation method using a microgrid model simulated in a realtime simulator from RTDS [10]. The test setup is illustrated in Fig. 6. On the left hand side, it is the microgrid model, which contains an energy storage system, a PV generation unit, a diesel generator, and a number of loads.

The microgrid is connected to the main grid during normal operating conditions, but in some cases, it could be unintentionally disconnected the from main grid due to events, e.g. faults. In such cases, it is important to accurately estimate the power imbalance size at the moment of sudden disconnection so that appropriate actions, e.g. generation dispatch or load shedding, can be taken to re-balance the system. In this example, the diesel generator is controlled using a droop-type governor [7], the PV inverter is controlled using the constant active and reactive power controllers [11], while the energy storage system is equipped with inertia emulation capability to enhance the frequency stability [12].

The diesel generator is rated at 3 MVA with an inertia constant of $2 \mathrm{~s}$. The energy storage unit is rated at 1 MVA and is controlled to emulate a machine with an inertia constant of $4 \mathrm{~s}$. In the studies, the simulation is conducted using the RTDS simulator and the simulated frequency and RoCoF data is recorded. The proposed event estimation algorithm is implemented in Simulink, which imports the recorded frequency and RoCoF data from RTDS simulation to estimate the event sizes. In an ultimate implementation, the event estimation algorithm will be implemented in a real-time controller platform and the estimation results can be used directly for control actions, which will be sent back to the RTDS for close-loop simulation.

Fig. 7 presents the simulation results using the microgrid model. Fig. 7.(a) shows the case where the inertia emulation is disabled. The microgrid is disconnected from the main network at $1 \mathrm{~s}$ with the demand greater than the local generation by $212 \mathrm{~kW}$. The measured maximum RoCoF magnitude is approximately $0.77 \mathrm{~Hz} / \mathrm{s}$. The estimated event size for both conventional and the proposed methods is approximately 176 

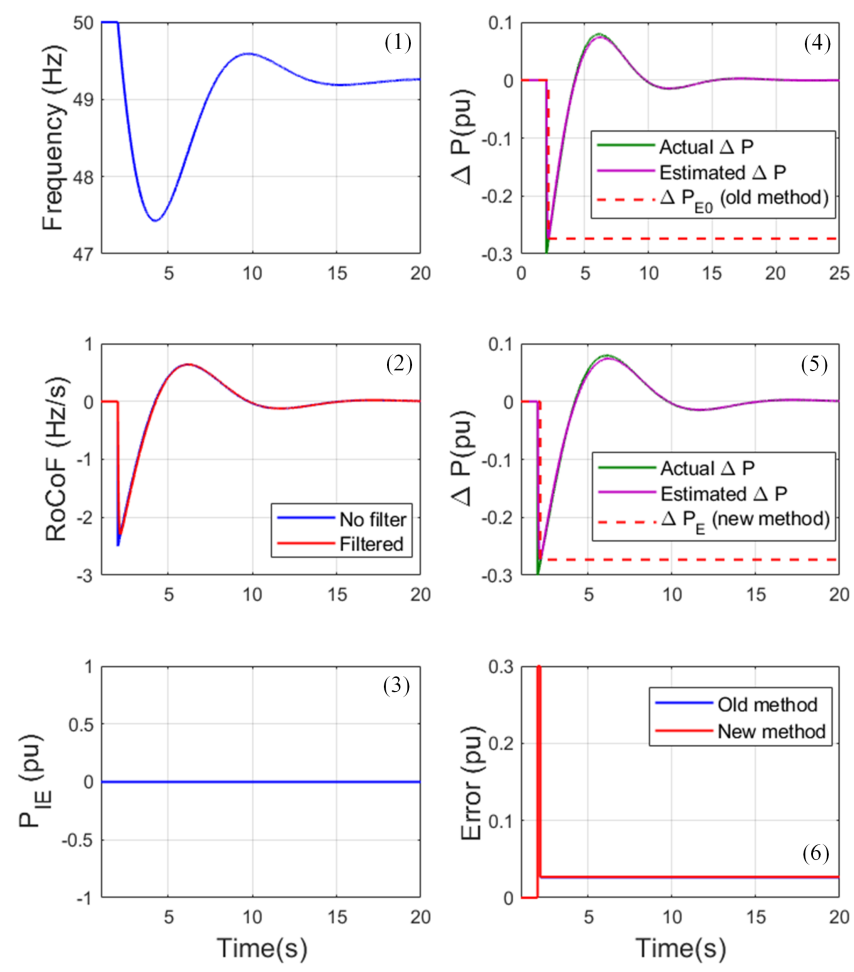

(a) Event size estimation when there is no inertia emulation
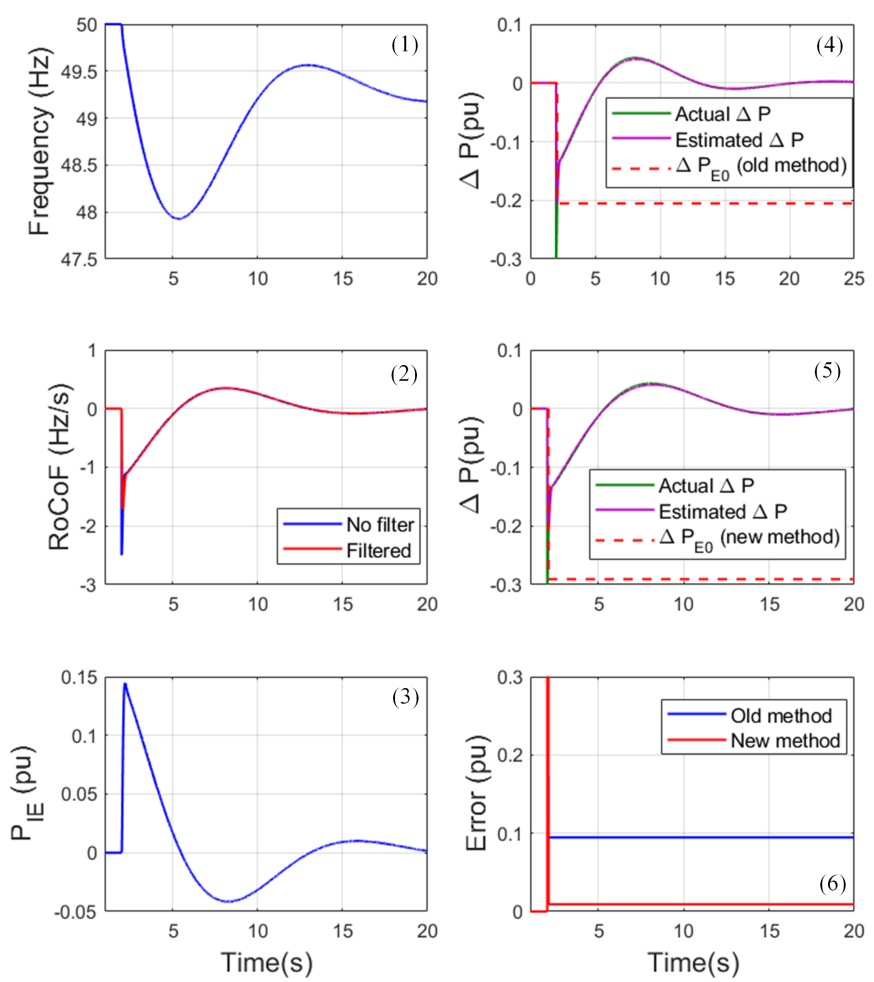

(b) Event size estimation when there is inertia emulation

Fig. 4. Simulation results using the analytical model

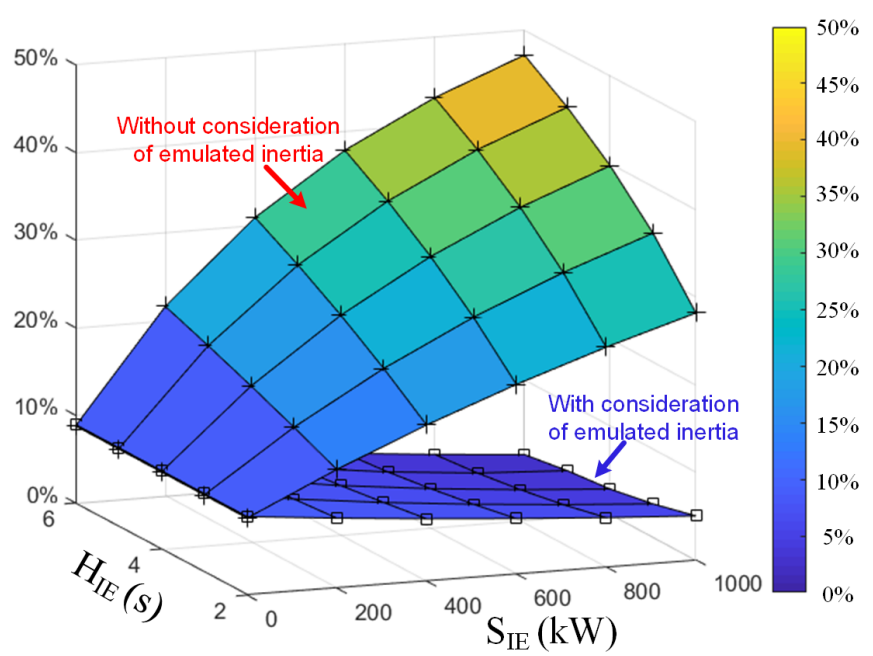

Fig. 5. Comparison of theoretical RoCoF and measured RoCoF during frequency disturbance

$\mathrm{kW}$. The error is mainly caused by the filtering of the RoCoF measurement to eliminate undesirable notices.

Fig. 7.(b) shows the results when the energy storage unit is enabled with inertia emulation capability. It can be seen that the measured maximum RoCoF becomes around $0.59 \mathrm{~Hz} / \mathrm{s}$ (as opposed to $0.77 \mathrm{~Hz} / \mathrm{s}$ in the case where no inertia emulation is used). This demonstrates the impact of inertia emulation on

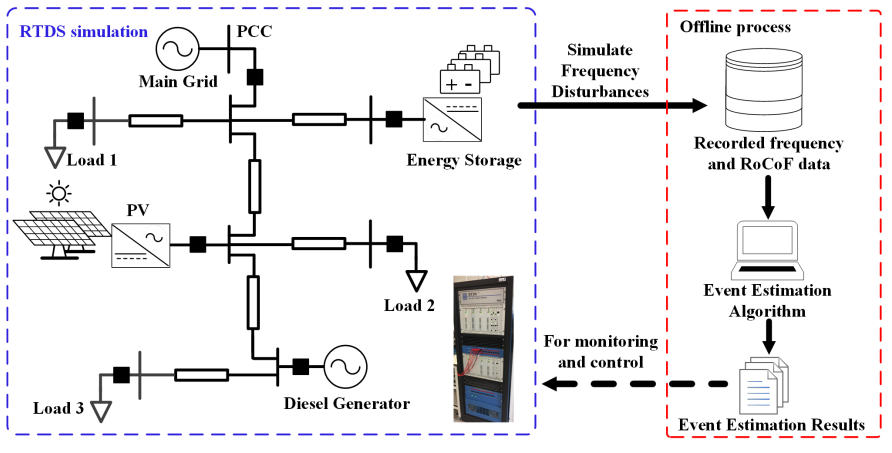

Fig. 6. Simulation results using the analytical model

the measured RoCoF with the same power imbalance event. Consequently, the estimation using the conventional method severely under-estimate the event size, which is estimated as approximately $133 \mathrm{~kW}$ as shown in the fourth plot. Using the proposed method, the estimation accuracy was significantly improved as shown in the fifth and sixth plot - the estimated size is $218 \mathrm{~kW}$, which is only $5 \mathrm{~kW}$ difference from the actual power imbalance. In contrast, the error is approximately 79 $\mathrm{kW}$ when using the conventional method.

\section{Conclusions}

This paper has investigated the impact of inertia emulation on the accuracy of power imbalance size estimation. It was shown that with the introduction of inertia emulation, the 

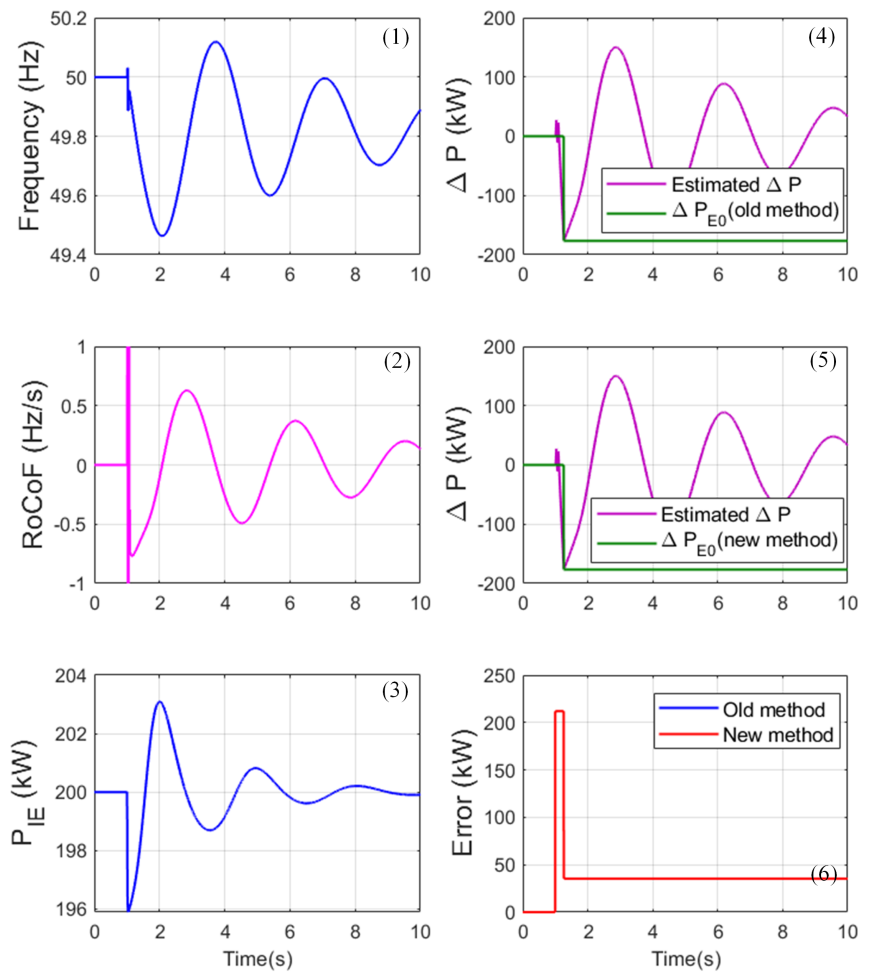

(a) Event size estimation when there is no inertia emulation
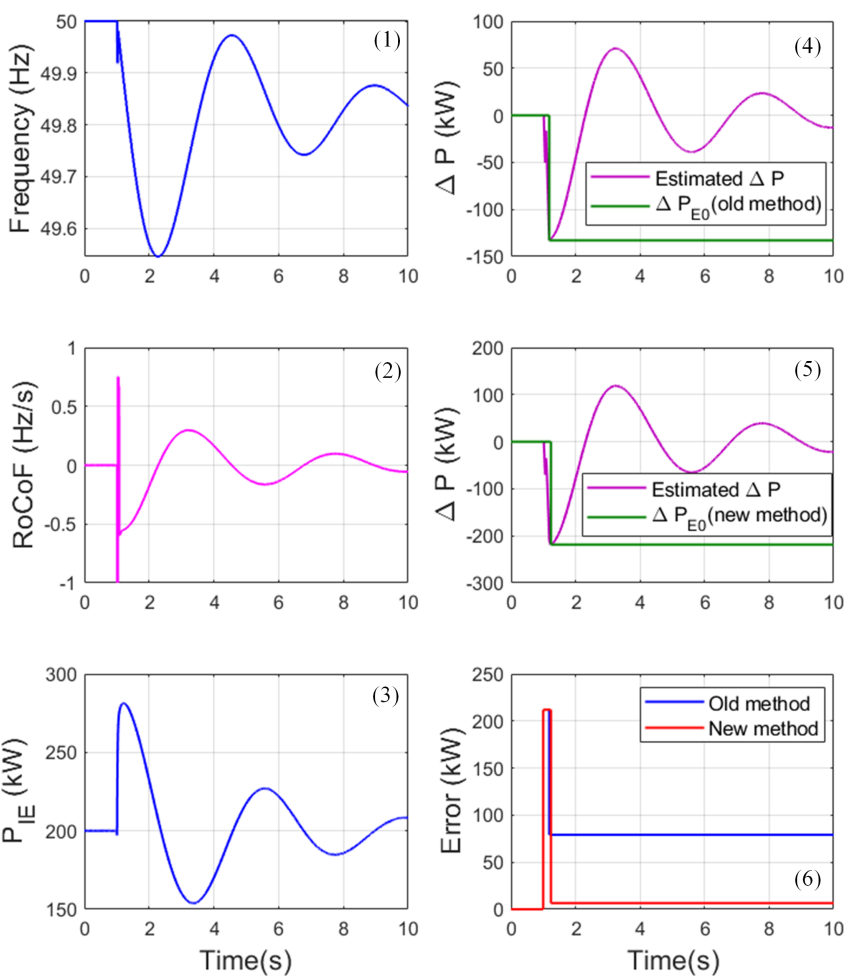

(b) Event size estimation when there is inertia emulation

Fig. 7. Simulation results using the microgrid model in RTDS

measured RoCoF could be affected and subsequently, the conventional method that is purely based on the measured RoCoF for event size estimation could lead to server errors. An analytical model has been presented and used to show that with the increasing capacity of inertia emulation units and the emulated inertia constant, the estimation error using the conventional method will also increase. The paper therefore proposed an approach that takes into account of the active power contribution from the inertia emulation in order to achieve more accurate estimation results. Cases studies using both analytical and power system models to have been presented, which demonstrate that the event size estimation accuracy can be significantly improved using the proposed method, which offers an promising solution for future control systems that requires the estimation of event size for decision making.

Future work will focus on the continued development of the proposed event estimation algorithm so that it can be used in a large system with different types of generation units providing inertia emulation functions. Furthermore, the algorithm will also be implemented and tested in a real-time platform so that the event estimation results can be used in real time for various monitoring and control purposes.

\section{REFERENCES}

[1] Q. Hong, M. Nedd, S. Norris, I. Abdulhadi, M. Karimi, V. Terzija, B. Marshall, K. Bell, and C. Booth, "Fast frequency response for effective frequency control in power systems with low inertia," The Journal of Engineering, vol. 2019, no. 16, pp. 1696-1702, 2019.
[2] V. Terzija, "Adaptive underfrequency load shedding based on the magnitude of the disturbance estimation," IEEE Transactions on Power Systems, vol. 21, no. 3, pp. 1260-1266, Aug 2006.

[3] V. Terzija, D. Cai, and M. Kayikci, "Power imbalance estimation in distribution networks with renewable energy resources," in CIRED 2009 - The 20th International Conference and Exhibition on Electricity Distribution - Part 2, June 2009, pp. 1-14.

[4] R. Azizipanah-Abarghooee, M. Malekpour, M. Paolone, and V. Terzija, "A new approach to the online estimation of the loss of generation size in power systems," IEEE Transactions on Power Systems, vol. 34, no. 3, pp. 2103-2113, May 2019.

[5] National Grid, "System Operability Framework 2016," Tech. Rep., 2016.

[6] J. Zhu, C. D. Booth, G. P. Adam, A. J. Roscoe, and C. G. Bright, "Inertia emulation control strategy for vsc-hvdc transmission systems," IEEE Transactions on Power Systems, vol. 28, no. 2, pp. 1277-1287, May 2013.

[7] P. Kundur, Power System Stability and Control. McGraw-Hill, 1993.

[8] P. S. Wright, P. N. Davis, K. Johnstone, G. Rietveld, and A. J. Roscoe, "Field measurement of frequency and rocof in the presence of phase steps," IEEE Transactions on Instrumentation and Measurement, vol. 68, no. 6, pp. 1688-1695, June 2019.

[9] P. M. Anderson and M. Mirheydar, "A low-order system frequency response model," IEEE Transactions on Power Systems, vol. 5, no. 3, pp. 720-729, Aug 1990.

[10] RTDS Technologies, Real Timme Digital Simulation for the Power Industry Manual Set.

[11] F. Blaabjerg, R. Teodorescu, M. Liserre, and A. V. Timbus, "Overview of control and grid synchronization for distributed power generation systems," IEEE Transactions on Industrial Electronics, vol. 53, no. 5, pp. 1398-1409, Oct 2006.

[12] T. Kerdphol, F. S. Rahman, and Y. Mitani, "Virtual inertia control application to enhance frequency stability of interconnected power systems with high renewable energy penetration," Energies, vol. 11, no. $4,2018$. 